\title{
Clinico-Radiological Diagnosis of Isolated Congenital Esophageal Stenosis in a Preterm Neonate
}

\author{
Minakshi Sham ${ }^{1}$ \\ ${ }^{1}$ Department of Paediatric Surgery, B. J. Medical College, Pune, \\ Maharashtra, India \\ Eur J Pediatr Surg Rep 2013;1:27-29.
}

\begin{abstract}
Address for correspondence Minakshi Sham, MCh, G-101 Sudarshan Apartments, Behind Foodworld, Karvenagar, Pune 411052, Maharashtra State, India (e-mail: minakshi_dr@rediffmail.com).
\end{abstract}

\begin{abstract}
Keywords

- congenital esophageal stenosis

(CES)

- esophageal atresia

(EA)

- preterm low birth weight (LBW) neonate

- primary resection anastomosis

- radiological diagnosis

A 2-day-old preterm female neonate weighing $1.6 \mathrm{~kg}$ and having excessive frothing from mouth was investigated for suspected esophageal atresia and tracheoesophageal fistula. X-ray findings of an unusually low-ending upper pouch (up to $T_{8}$ level) and the absence of gas in abdomen lead to suspicion of an unusual variety of esophageal atresia. Hence unlike the usual management of pure esophageal atresia, in terms of esophagostomy and gastrostomy in neonatal period, right thoracotomy was performed allowing successful primary anastomosis. A high index of suspicion on the basis of radiological picture led to early diagnosis of a rare anomaly like congenital esophageal stenosis and successful management of this low birth weight baby.
\end{abstract}

\section{Introduction}

Congenital esophageal stenosis (CES) is a rare, yet significant entity because of its clinical manifestation and its association with tracheoesophageal fistula (TEF) or esophageal atresia (EA). ${ }^{1}$ The incidence of CES is estimated at 1 in 25,000 to 50,000 live births. ${ }^{2}$ There is associated EA in one-third of the cases; the remainder are classified as isolated CES. ${ }^{2}$ Three histopathologic types of CES have been described in the literature. Management options include dilatation, endoscopic excision, or segmental resection. Our case, a 2-day old preterm female neonate weighing $1.6 \mathrm{~kg}$, underwent primary excision of the stenotic segment and esophageal reanastomosis with ultimate healing of the anastomosis. High index of suspicion of mid/lower EA on the basis of Xray findings led us decide upon thoracotomy rather than performing stomas and a staged surgery in this preterm low birth weight neonate.

\section{Case History}

A 2-day-old female neonate, weighing $1.6 \mathrm{~kg}$ was brought to us with history of excessive frothing from the mouth. She was second born, vaginally delivered at a gestational age of 35 weeks. The pregnancy and delivery were uncomplicated. The antenatal ultrasonography was reported to be normal. On examination, thick mucoid secretions were emanating from the mouth. The baby had moderate respiratory distress, with respiratory rate of $80 / \mathrm{min}$. Her cry, tone, and activity were normal. The abdomen was scaphoid. Anal opening, external genitals, and the spine were normal. Baby had passed meconium. There were no stigmata of any other congenital anomaly. X-ray chest with gentle pressure on the stiff (10 Fr) red rubber catheter inserted through mouth showed upper pouch almost upto $T_{8}$ level ( - Fig. 1 ). There was no gas visible in the abdomen and pelvis (gasless abdomen). Ultrasonography of the abdomen was normal.

(c) 2013 Georg Thieme Verlag KG Stuttgart · New York
DOI http://dx.doi.org/ 10.1055/s-0033-1345279. ISSN 2194-7619. 


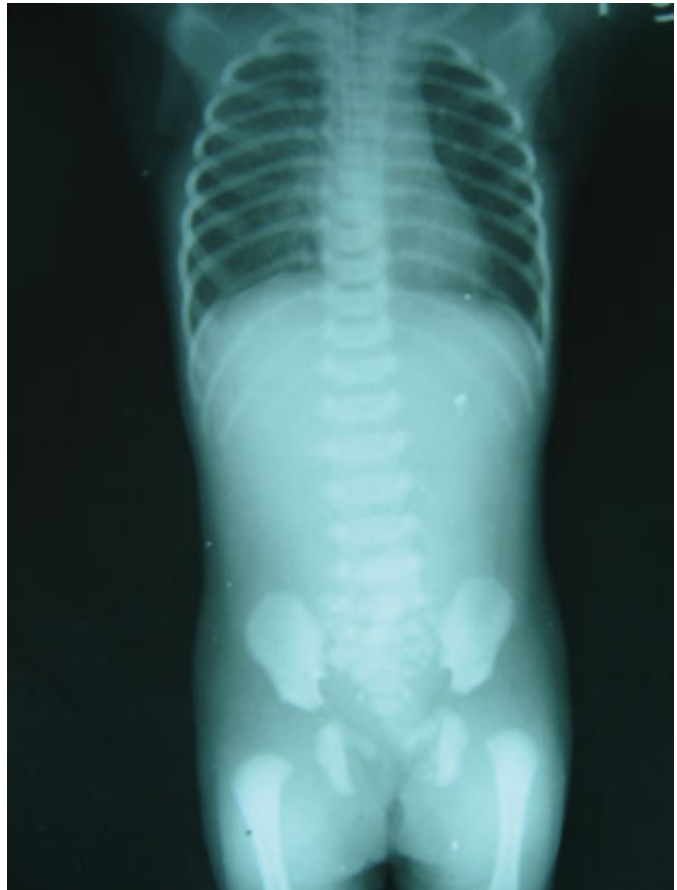

Fig. 1 Chest $\mathrm{X}$-ray showing upper pouch ending at $\mathrm{T}_{8}$. Also note the absence of gas in the abdomen and pelvis.

Suspecting an unusual type of atresia of the esophagus, we chose to surgically intervene. A right thoracotomy was done through sixth intercostal space (transpleural approach). The upper pouch was dilated to $1.5-\mathrm{cm}$ diameter and in-continuity with the lower pouch (-Fig. 2). The lower pouch was grossly thickened with wall thickness of 4 to $5 \mathrm{~mm}$. There was stenotic segment of approximately 4 to $5 \mathrm{~mm}$ between the two pouches, which was excised. No fistula was identified. Spatulation of the lower pouch was done to match the diameter of the upper pouch. A single layer, end to oblique esophago-esophageal anastomosis was done using 5-0 Polyglactin over a nasally placed $6 \mathrm{Fr}$ infant feeding tube. There was no tension at the suture line. The thoracic cavity was

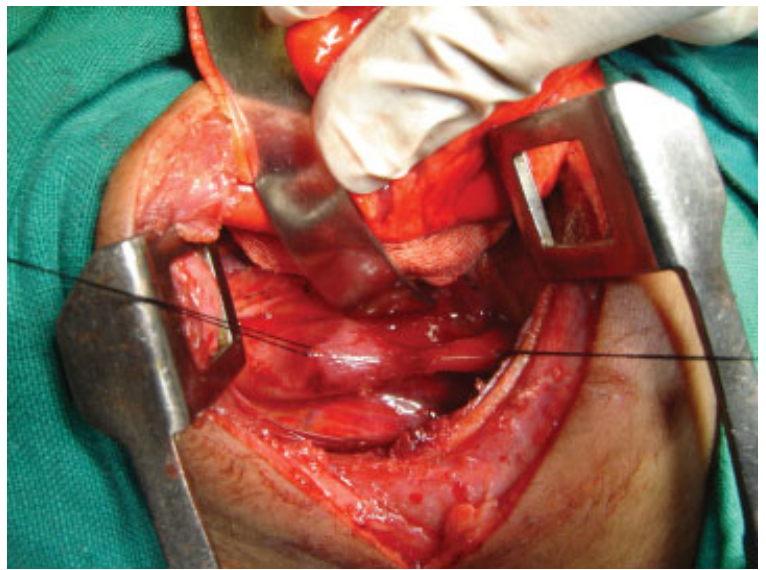

Fig. 2 Operative photograph showing dilated upper pouch, in-continuity with the thickened lower pouch. No fistula is noted.

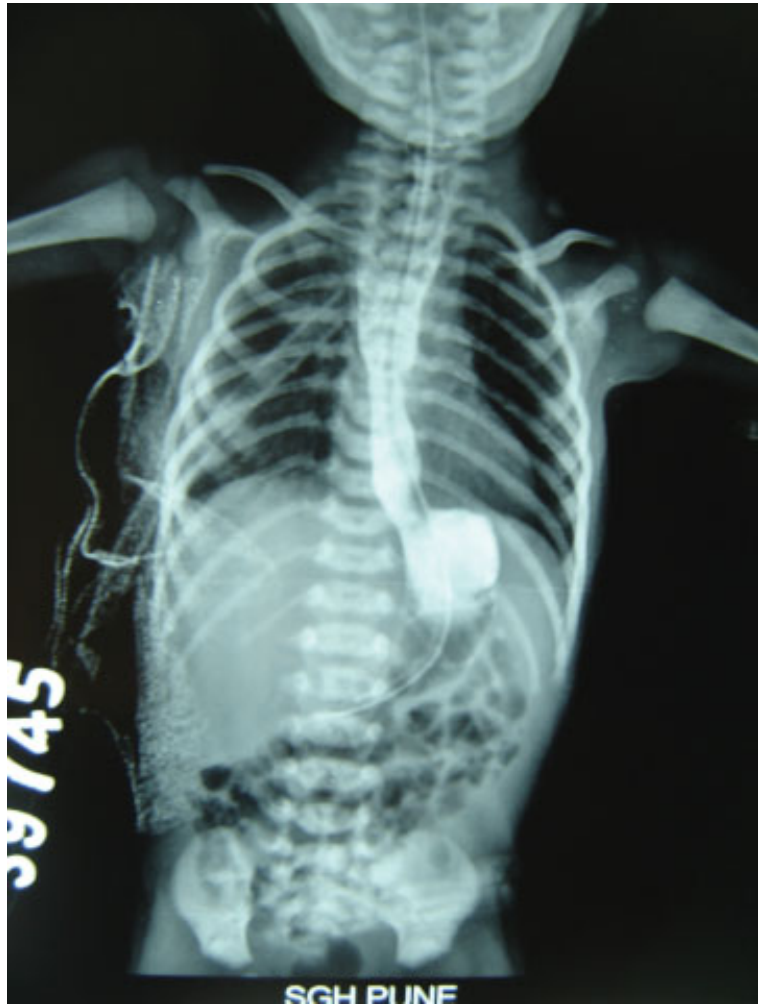

Fig. 3 Contrast study showing a widely patent anastomosis and no anastomotic leak.

drained by intercostal tube drainage, and the incision was closed in layers.

Postoperatively, intravenous antibiotics, regular chest physiotherapy, and normal saline nebulization were given. A radiocontrast study using water-soluble dye was done on the 7th postoperative day. There was no anastomotic leak and the contrast flowed freely across the anastomosis into the stomach within seconds (-Fig. 3). Feeds were started through the feeding tube initially and gradually advanced to full orals. The infant feeding tube was removed on 9th postoperative day. Intercostal drain was taken out on the 10th postoperative day. Overall, there was no complication.

The histopathology of the excised stenotic segment showed smooth muscle hypertrophy. Presently, at a followup age of 10 months, the baby is thriving well and has normal milestones for her age. Barium study at 10 months shows a widely patent anastomosis and absence of any gastroesophageal reflux.

\section{Discussion}

CES is a rare, yet significant entity because of its clinical manifestation and its association with TEF or EA (EA). ${ }^{1}$ In stenosis, there is obliteration of the esophageal lumen. Stenosis results from abnormal rests of respiratory tissue in the esophageal wall or fibromuscular hypertrophy. As per the popularly used Gross classification, esophageal stenosis, that is, Type F of EA along with Types B, D, and G that are rarer varieties, together constitutes 3 to $5 \%$ of all EAs, thus defining the rarity of the condition. ${ }^{3,4}$ 
The clinical presentation of CES is varied, and a high index of suspicion is essential to arrive at an early diagnosis. ${ }^{5}$ Management depends on the type and severity of the stenosis. Stenosis due to tracheobronchial remnants requires segmental resection. Fibromuscular stenosis may respond to dilatation, but severe degrees of stenosis require segmental excision. Membranous diaphragms usually respond to dilatation or may require endoscopic excision. ${ }^{5}$

Three cases of CES of different anatomical varieties involving the lower third of esophagus have been described by Ramesh et al. ${ }^{5}$ They have done segmental resection anastomosis in two patients and dilatation for one child.

In this case, the history of excessive frothing from the mouth as well as radiological findings of the upper pouch extending upto $T_{8}$ level alongwith the absence of gas in the abdomen and pelvis led to suspicion of lesion in the lower third of esophagus. Suspecting underlying esophageal stenosis, decision was taken to perform a right thoracotomy. Intraoperatively, in case the lower pouch was found undeveloped/far off for a safe anastomosis, we had planned to pull up the stomach (primary pull through). Hence, we purposefully entered the thoracic cavity through the sixth intercostal space despite the usual entry for TEF through the fourth intercostal space. The upper and lower pouches were in-continuity. However, the real challenge was anastomosing the grossly thickened lower pouch to the grossly dilated $(1.5 \mathrm{~cm})$ upper pouch. Excision of the stenotic segment and adequate spatulation of the lower pouch to match the diameter of the upper pouch was done. As a result, almost an end-to-oblique, widely patent, tension-free, primary esophageal anastomosis could be achieved. Antireflux procedure was not considered necessary because we did not have to mobilize the lower pouch at all and its length was adequate.

Few other authors have previously described the management of CES by primary resection anastomosis. Murphy et $\mathrm{al}^{2}$ have described successful management of three patients with isolated CES, using limited resection anastomosis via a left thoracotomy. Their patients presented around 6 to 12 months of age, with feeding difficulty upon introduction of solids.
Initial hydrostatic dilatation was tried, but later on they had to resort to resection anastomosis because of failure of dilatation. Similar to our case, the stenoses were located in the distal third of esophagus, near its junction with the middle third. Amae et al also had done resection anastomosis in 11 of 14 patients who had initially undergone dilatation. ${ }^{6}$

The crux of management of our case was the decision making to plan thoracotomy and primary repair in the neonatal period. In the normal course of events, one would do esophagostomy and feeding gastrostomy in a neonate with (long gap) pure EA. In our case, we were sure that the upper pouch was pretty long, and hence we decided to take the risk and open the thoracic cavity. Here, the decision of performing thoracotomy was more crucial rather than the actual technical (operative) exercise. This case also highlights the importance of accurate interpretation of a well-taken chest X-ray with a stiff (10 Fr) rubber catheter in situ for diagnosis and management of a very rare variety of EA.

\section{Conflict of Interest}

None

\section{References}

1 Vasudevan SA, Kerendi F, Lee H, Ricketts RR. Management of congenital esophageal stenosis. J Pediatr Surg 2002;37(7):10241026

2 Murphy SG, Yazbeck S, Russo P. Isolated congenital esophageal stenosis. J Pediatr Surg 1995;30(8):1238-1241

3 Gross RE. Atresia of the esophagus. In: Gross RE, ed. Surgery of Infancy and Childhood. Philadelphia, PA: Saunders; 1953:75

4 Gupta DK, Dave S. Esophageal atresia. In: Gupta DK, ed. Text Book of Neonatal Surgery. 1st ed. New Delhi, India: Modern Publishers; 2000:317-335

5 Ramesh JC, Ramanujam TM, Jayaram G. Congenital esophageal stenosis: report of three cases, literature review, and a proposed classification. Pediatr Surg Int 2001;17(2-3):188-192

6 Amae S, Nio M, Kamiyama T, et al. Clinical characteristics and management of congenital esophageal stenosis: a report on 14 cases. J Pediatr Surg 2003;38(4):565-570 